\title{
Heavy Hydrogen*
}

\section{By the Right HoN. LoRd RUtherford, O.M., F.R.S.}

$\mathrm{I}^{\mathrm{N}}$ the history of physical science, it is a commonplace that a new discovery which at first appears to be of purely scientific interest, ultimately, within a period of twenty years or more, is found to have useful practical applications. This is well illustrated by the discovery of the rare gases in the atmosphere, neon and argon, which are now used in quantity for industrial purposes. The fundamental discovery in 1919 of the isotopic constitution of the majority of our elements, so largely due to Aston, at first sight appeared to be of purely scientific significance, but it may ultimately have wide practical consequences in many directions.

It is scarcely necessary to discuss in detail the history of the discovery and separation of heavy hydrogen, in which scientific workers in the United States have taken such a leading part. The proof that oxygen was not a simple element but contained two isotopes in small quantity of masses 17 and 18, indicated that there was a small discrepancy of about two parts in 10,000 between the measurements of the relative masses of hydrogen and oxygen found by Aston and those found by direct physical and chemical methods. Birge and Mendel suggested that this discrepancy might be due to the presence of an isotope of mass 2 present in ordinary hydrogen. This gave the necessary impetus to Urey, Brickwedder and Murphy to test whether the presence of $\mathrm{H}^{2}$ could be detected by direct optical methods. The experiments were successful in showing a small trace of $\mathrm{H}^{2}$, estimated initially at about $\mathrm{I}$ in 4,000 of the $\mathrm{H}^{1}$ isotope. The wave-length of the $\alpha$ line of $\mathrm{H}^{2}$ was found to be 1.79 A. greater than for $\mathrm{H}^{1}$-a result agreeing closely with the theoretical value to be expected for an isotope of hydrogen of mass 2 . The mass of the new isotope was directly measured by Bainbridge, using a modified type of mass-spectrograph, and found to be 2.0136 , slightly less than the weight of the ordinary hydrogen molecule, $2 \cdot 0156$, in terms of $\mathrm{O}=16$.

We have no definite evidence of the exact constitution of $\mathrm{H}^{2}$, whether it should be regarded as a simple entity or built up of two or more constituents. It was at first natural to suppose that the $\mathrm{H}^{2}$ nucleus might be made up of two protons and a negative electron, but the subsequent discovery of the neutron indicated that it might rather be a close combination of a neutron and a proton. Taking Chadwick's value of the mass of the neutron as 1.0067 , the sum of the masses of the proton and neutron is $2 \cdot 0145$, while the mass of the $\mathrm{H}^{2}$ nucleus is slightly less, $2 \cdot 0136$, indicating that the binding energy of the neutron-proton combination is less than one million volts. If this be the case, it is to be expected that the $\mathrm{H}^{2}$ nucleus

"Address delivered in opening a discussion on "Heavy Hydrogen" at the Royal Society on December 14. should be broken up by collision with a swift -particle. In conjunction with Mr. Kempton, I have made experiments to test this, but have been unable to detect with certainty the presence of any neutrons when heavy water was bombarded by $\alpha$-particles from polonium. The number of neutrons, if any, was certainly less than 1 per cent of the number of neutrons released from a sheet of beryllium under the same conditions. If the disruption of $\mathrm{H}^{2}$ with an emission of a neutron occurs, it must happen very rarely compared with the number of violent collisions between the $\alpha$-particles and the $\mathrm{H}^{2}$ nucleus.

It is interesting to note here a suggestion made by Lawrence. He found in his experiments on the bombardment of matter by high-speed $\mathrm{H}^{2}$ ions that a group of protons of nearly the same speed was released from a number of elements. In explanation, he suggested that the $\mathrm{H}^{2}$ nucleus broke up into a neutron and proton either in the bombarded nucleus or in the strong field in its neighbourhood. For the conservation of energy to hold, it is necessary to suppose that the mass of the neutron is much lower than that found by Chadwick, namely, 1.0006 instead of 1.0067. On this view, the $\mathrm{H}^{2}$ nucleus contains a store of energy corresponding to about five million volts, and this is occasionally released in nuclear collisions. Further experiments are required to test the validity of this idea.

It was of interest to me also to examine whether the fields of force near the $\mathrm{H}^{1}$ and $\mathrm{H}^{2}$ nuclei are the same. This was tested by comparing the distribution with the velocity of the recoil $\mathrm{H}^{1}$ and $\mathrm{H}^{2}$ atoms when $\alpha$-particles pass through ordinary and heavy hydrogen respectively. While the recoil $\mathrm{H}^{2}$ particles travel, as is to be expected, slightly farther than the $\mathrm{H}^{1}$ particles, to a first approximation the number and distribution of the recoil atoms were about the same in the two cases. Since in a close collision the $\alpha$-particles and the $\mathrm{H}^{2}$ nucleus approach within $10^{-12} \mathrm{~cm}$. of each other, these results indicate that the scattering fields are sensibly the same for $\mathrm{H}^{1}$ and $\mathrm{H}^{2}$ nuclei, even up to these very small distances.

Some success in concentrating $\mathrm{H}^{2}$ was initially obtained by fractionating liquid hydrogen. Washburn and Urey noted that there was a greater concentration of $\mathrm{H}^{2}$ in old electrolytic cells and found the $\mathrm{H}^{2}$ was rapidly enriched in the residues after electrolysis. This general method was first used on a large scale by Lewis and Macdonald of the University of California in order to obtain a concentrated preparation of heavy water. By this method they have obtained quantities of heavy water of the order of several hundred cubic centimetres practically in a pure state. It has been concluded that one atom of $\mathrm{H}^{2}$ is normally present with 6,500 atoms of ordinary hydrogen. Lewis 
and his collaborators find that the density of this new water is about 11 per cent higher than that of ordinary water, while its freezing point is $3 \cdot 8^{\circ} \mathrm{C}$. and its boiling point $101 \cdot 42^{\circ} \mathrm{C}$. The maximum density is found to occur at $11 \cdot 6^{\circ} \mathrm{C}$. instead of at $4^{\circ} \mathrm{C}$. as in normal water.

It is of interest also to refer to another means of concentration carried out by utilising pure diffusion methods. Prof. Hertz informs me that he has been able to obtain the new isotopes in small quantity in a pure state by applying to ordinary hydrogen the elaborate diffusion method worked out by him. He states that he has obtained heavy hydrogen so pure that he has been unable to detect in its spectrum the $\alpha$-line of ordinary hydrogen. Dr. P. Harteck, working in the Cavendish Laboratory, has been responsible for a preparation of about 25 c.c. of the new heavy water for use in experiments on the transformation of matter.

It is obvious that this new discovery opens up a wide and important field of work. On account of its greater mass, it is to be expected that the rate of diffusion and the rate of chemical reaction will differ when $\mathrm{H}^{2}$ is substituted for $\mathrm{H}^{1}$, while the compounds formed with the new isotope are to be expected in some cases to exhibit rather different properties from the normal hydrogen compounds. Similarly, this new discovery opens up interesting questions on the effect of heavy water in altering the normal physical and chemical processes in animal and plant life. A certain amount of information is already available in this interesting field of inquiry.

There is one question of much interest to me to which I should like to refer, namely, the use of $\mathrm{H}^{2}$ nuclei as swift projectiles for studying the transformation of the elements. It was a happy coincidence that when Prof. Lewis had prepared concentrated samples of $\mathrm{H}^{2}$, Prof. Lawrence of the same University had in working order his ingenious apparatus for obtaining high-speed ions corresponding to more than a million volts in energy. Lawrence found that the high-speed $\mathrm{H}^{2}$ ions were much more effective in many cases than protons of equal energy in causing transformations of new kinds in a number of elements. For example, when lithium is bombarded with $\mathrm{H}^{2}$ ions, $\alpha$ particles are ejected with a speed considerably greater than the swiftest $\alpha$-particle from radioactive substances. It is now clear that an $\mathrm{H}^{2}$ particle occasionally enters the nucleus of lithium of mass 6 , and the resulting nucleus then breaks up into two $\alpha$-particles, escaping in nearly opposite directions. The correctness of this view is well shown by the Wilson chamber photographs of the tracks of the $\alpha$-particles obtained by Dee and Walton.

The action of $\mathrm{H}^{2}$ on the lithium isotope of mass 7 is even more complicated, for Oliphant and I have observed that $\alpha$-particles are liberated over a wide range of velocities. In this case, it seems that the capture of $\mathrm{H}^{2}$ by the lithium nucleus of mass 7 results in the break up of the system into two $\alpha$-particles and a neutron. We estimate that the maximum energy of the ejected neutron may be as great as fifteen million volts. We have confirmed this conclusion by finding that neutrons can be detected in numbers corresponding to this mode of transformation using $\mathrm{H}^{2}$ particles of energy about 200,000 volts. Lauritsen found that a copious supply of neutrons could be obtained by bombarding beryllium with $\mathrm{H}^{2}$ particles, while Lawrence obtained large numbers from lithium with very fast $\mathrm{H}^{2}$ particles, but he inclines to believe that most of the neutrons observed in his experiments arise from the break up of the $\mathrm{H}^{2}$ nucleus into a neutron and a proton.

As already mentioned, Lawrence has observed that $\mathrm{H}^{2}$ bombardment gives rise to one or more groups of fast protons from a number of elements. These observations have been confirmed by Cockcroft and Walton for several light elements such as lithium, carbon and iron, using $\mathrm{H}^{2}$ particles of energy about 500,000 volts, but they have failed to observe proton groups from copper and gold. In general, it appears that the $\mathrm{H}^{2}$ particle is remarkably effective in causing the transformation of many elements, resulting in a number of cases in the liberation of $\alpha$-particles as well as protons and neutrons. There can be no doubt that this new projectile, as well as the proton, will prove of great service in studying the processes which take place in the transformation of the elements, and this will give further important information on the structure of nuclei.

It is obvious that this new isotope, which can be obtained in reasonable quantity in a pure state so easily, will prove of such great importance to science that it is desirable to give it a definite name. Urey has proposed the name 'deuterium' for the new isotope. It is important also that an appropriate title should be given to the $\mathrm{H}^{2}$ nucleus, not only as a projectile for atomic transmutations, but as a possible constituent of atomic nuclei. Lewis has suggested the name 'deuton' or 'deuteron' for this nucleus. While we all realise that the first discoverer has a strong claim in suggesting an appropriate name for a new substance, the question of a suitable nomenclature is in this case of such general importance to scientific men that it deserves very careful consideration.

While the name 'deuton' is in some ways suitable, it has for me the objection that it is liable in the spoken word to be confused with neutron, and this difficulty is accentuated by the recent discovery that neutrons are liberated in many cases from elements bombarded by deutons. In consultation with some of my physical and chemical colleagues, some time before these names were announced, the name 'diplogen' ( $\delta \iota \pi \lambda_{\circ} \hat{\imath}$, double) for heavy hydrogen, and 'diplon' for the nucleus seemed to meet with some favour. Whatever view may eventually be taken on this question, it is important that the new isotope should have a definite symbol allotted to it, and the symbol ' $D$ ' seems appropriate. 